\title{
Evolutionary Optimisation of JPEG2000 Part 2 Wavelet Packet Structures for Polar Iris Image Compression
}

\author{
Jutta Hämmerle-Uhl, Michael Karnutsch, and Andreas Uhl \\ Multimedia Signal Processing and Security Lab \\ Department of Computer Sciences, University of Salzburg, Austria \\ uhl@cosy.sbg.ac.at
}

\begin{abstract}
The impact of using evolutionary optimised wavelet subband stuctures as allowed in JPEG2000 Part 2 in polar iris image compression is investigated. The recognition performance of two different feature extraction schemes applied to correspondingly compressed images is compared to the usage of the dyadic decomposition structure of JPEG2000 Part 1 in the compression stage. Recognition performance is significantly improved, provided that the image set used in evolutionary optimisation and actual application is identical. Generalisation to different settings (individuals, sample acquisition conditions, feature extraction techniques) is found to be low.
\end{abstract}

\section{Introduction}

The International Organization for Standardization (ISO) specifies iris biometric data to be recorded and stored in (raw) image form (ISO/IEC FDIS 19794-6), rather than in extracted templates (e.g. iris-codes). On the one hand, such deployments benefit from future improvements (e.g. in feature extraction stage) which can be easily incorporated,without re-enrollment of registered users. On the other hand, since biometric templates may depend on patent-registered algorithms, databases of raw images enable more interoperability and vendor neutrality [1, 2]. These facts motivate detailed investigations and optimisations of image compression on iris biometrics in order to provide an efficient storage and rapid transmission of biometric records. Furthermore, the application of low- powered mobile sensors for image acquisition, e.g. mobile phones, raises the need for reducing the amount of transmitted data. There are two options in iris recognition: the acquired sample data can be compressed and transfered as it has been obtained by the sensor (termed "rectilinear images"), or the iris texture strip as obtained from prior segmentation and log-polar mapping (termed "polar iris image") may be compressed and transfered. The second option obviously trades off the higher computational cost at the sensor (segmentation + compression) for a minimisation of the transfered data amount.

The certainly most relevant standard for compressing image data relevant in biometric systems is the ISO/IEC 19794 standard on Biometric Data Interchange Formats where only JPEG2000 is included for lossy compression. In literature on compressing iris imagery, rectilinear [1, 2, 3] as well as polar [2, 4] iris sample data has been considered. With respect to employed compression technology, we find JPEG [1, 2] JPEG2000 [1, 2, 3, 4], JPEG XR, and other general purpose compression techniques [2] being investigated.

J. Ruiz-Shulcloper and G. Sanniti di Baja (Eds.): CIARP 2013, Part I, LNCS 8258, pp. 391-398, 2013.

(c) Springer-Verlag Berlin Heidelberg 2013 
In biometrics, wavelet packet based image compression schemes have been applied before in the area of fingerprint recognition (e.g. [5, 6]) due to the high frequency nature of the ridge and valley pattern in fingerprint imagery. Eventually, similar to fingerprint images, image features important for iris template matching might reside in high or mid frequency parts of the iris texture, which could be represented better by adapted wavelet packet structures.

In this work, we employ wavelet packet decomposition structures for the compression of polar iris images using JPEG2000 Part 2 technology. Recent work [7] showed that common subband structure selection selection strategies including rate-distortion optimising ones are not very successful as compared to the dyadic decomposition scheme (defined in the Part 1 of the JPEG2000 standard suite). Therefore, in this work, evolutionary optimisation is applied to select subband structures where recognition performance is used as optimisation criterion (while much simpler criteria are used in [7]). In Section 2 , we review the use of wavelet packets in JPEG2000 and discuss various subband structure selection strategies inlcuding the evolutionary approach used in this paper. Section 3 provides experimental results for two different iris recognition schemes while Section 4 concludes the paper.

\section{Wavelet Packet Selection and JPEG2000}

The use of adapted wavelet packet bases for image compression purposes has been subject to investigation since the introduction of the first adaptation technique called "best basis algorithm". Due to the high number of wavelet packet bases ( $w p b$ ) (i.e., for a decomposition depth $d$, the number is $Q_{d}=Q_{d-1}^{4}+1$ with $Q_{0}=1$ ), exhaustive search is infeasible which has lead to the development of various $w p b$ selection strategies. The employment of rate-distortion optimization criteria for $w p b$ subband structure selection has been first demonstrated for classical wavelet image coding schemes, but has been extended later to zero-tree based compression algorithms [8] and to JPEG2000 in recent work [9]. While JPEG2000 Part 1 is restricted to the pyramidal wavelet transform (fixed dyadic decomposition), JPEG2000 Part 2 facilitates the use of more general wavelet packet subband structures [10] (besides the specification of user-defined wavelet filters and other advanced coding options).

All the so far described $w p b$ selection schemes have failed to significantly improve iris recognition performance as compared to dyadic decomposition [7]. Therefore, we want to directly optimise recognition performance instead of optimising certain cost functions usually targeted towards rate/distortion performance. However, common $w p b$ selection schemes rely on the independent evaluation of cost functions on single $w p b$ subbands, which is not possible when recognition performance of a certain $w p b$ has to be assessed. In earlier work [11], we have used genetic algorithms to assess the degree of optimality and to further optimize $w p b$ subband structues. This approach is adopted for the present study where the fitness function of the evolutionary approach rating a single $w p b$ is set to be a parameter describing recognition performance after compressing the data to JPEG2000 format using the corresponding $w p b$, i.e. the equal error rate (EER).

Genetic algorithms are random search procedures guided by evolutionary principles suited for vast search spaces, where parameter optimisation problems need to be solved. 
A key issue to apply the generic approach to the $w p b$ subband structure selection task is to find a suitable representation of the $w p b$ and to adapt genetic operators to the $w p b$ tree structures [11]. The $w p b$ can also be considered as a quadtree which needs to be transformed into a "flat" representation: in adopting principles of the heap sort algorithm, a string $b$ of finite length $L$ over a binary alphabet $\{0,1\}$ is used. With a particular bit $k$ set to 1 , the corresponding subband is decomposed, otherwise the index $\mathrm{k}$ is set to 0 and the decomposition stops in this part of the tree. Determining the indices for the corresponding subbands is accomplished by $k_{m}^{\prime}=4 * k+m, 1 \leq m \leq 4$. After having chosen two particular individuals for being candidates for the next generation, a kind of genetical material interchange is performed. Classical one- or two-point crossover operators cannot be applied since in general, the resulting bitstrings do not correspond to valid $w p b$. Therefore, sub-tree based crossover has been introduced [11] which exchanges sub-trees and maintains a valid tree structure. Other genetic operators like mutation and selection can be applied in a standard manner. For selection, we apply roulette wheel selection (where the probability of an individual $i$ for being chosen to be a candicate for the next generation is $p_{i}=f_{i} / \sum_{j=1}^{N} f_{j} ; f_{i}$ is the fitness value of the individual $i$ and is $N$ the total number of individuals per generation) as well as tournament selection (where the best $m$ out of $k$ randomly chosen individuals are selected for the next generation).

\section{Experiments}

\subsection{Experimental Settings}

As sample data, we use the public CASIA V3 Interval database consisting of 2639 images from 391 eye classes with $320 \times 280$ pixels and eight-bit grey value. These are extracted into polar iris images with $512 \times 64$ pixels, which act as the base for the following compression and subsequent iris recognition algorithms. For the evolutionary optimisation, the first 59 classes (555 images) were used, while the remaining data is used for intra-database verification. For cross-database verification, the first 555 images (i.e. 111 classes) of the IITD Iris Database version 1.0 are used, polar iris images are extracted into the same size.

Experimental results with respect to JPEG2000 Part 1 \& 2 compression have been generated using a custom implementation of $w p b$ selection strategies based on the JJ2000 reference implementation [9]. Bitrates 0.2bpp, 0.4bpp, 0.8bpp and 1.5bpp are considered. Both templates involved in biometric matching, the one generated from the sample data and the one from the database, are derived from images compressed to the same bitrate.

It is crucial to assess the effects of compressing iris samples using different iris recognition schemes since it can be expected that different feature extraction strategies will react differently when being confronted with compression artefacts and reduced image quality in general. We use custom implementations of two feature extraction techniques (for a description of our implementation of preprocessing, feature extraction, and matching see [2]). Both implementations are available in USIT (University of Salzburg Iris-Toolkit at http: / / wavelab.at. The first scheme has been developed by Ko 
et al. [12] and extracts spatial domain features, while the second approach has been designed by Monro et al. [13] and relies on DCT-derived features.

Evolutionary optimisation is started with 50 initial individuals $(w p b)$ in the first generation which are derived from results using techniques in [7]. After determining the fitness values (difference to the EER, which the dyadic decomposition at depth three achieves), either roulette wheel selection or tournament selection (with $k=5$ and $m=2$ ) is applied. Subsequently, tree crossover is repeatedly applied to two selected $w p b$ in order to generate two new individuals for the next generation and finally, mutation is applied to each bit in the $w p b$ representation of the new generation with a probability of 0.01 . This procedure is repeated over 50 generations.

In case of cross-bitrate optimisation (i.e. searching for $w p b$ superior to the dyadic case for more then just a single bitrate), the $w p b$ fitness is the number of bitrates, at which the particular $w p b$ outperforms the dyadic case, and as a second value either the mean or the standard deviation of the relative EER for the bitrates $(0.2 \mathrm{bpp}, 0.4 \mathrm{bpp}$, $0.8 \mathrm{bpp}, 1.5 \mathrm{bpp})$ is considered. In this case, only roulette wheel selection is applied.

\subsection{Experimental Results}

Figs. 1 and 2 are meant for illustrating the evolutionary process. On the $\mathrm{x}$-axis, the 50 generations are shown whereas on the $y$-axis the fitness values are given (a point in the plot corresponds to the value of a single individual $-w p b$ ). Below the two graphs (left: roulette wheel selection, right: tournament selection) three $w p b$ are shown: the one corresponding to the "best" $w p b$ in the initial generation in the middle, and the final "winning" individuals of the last respective generations left and right to it.

When considering the EER of the two recognition schemes under JPEG2000 Part 1 compression for the four bitrates $0.2 \mathrm{bpp}, 0.4 \mathrm{bpp}, 0.8 \mathrm{bpp}$, and $1.5 \mathrm{bpp}$ (i.e. $(E E R(K o)=$

roulette wheel selection

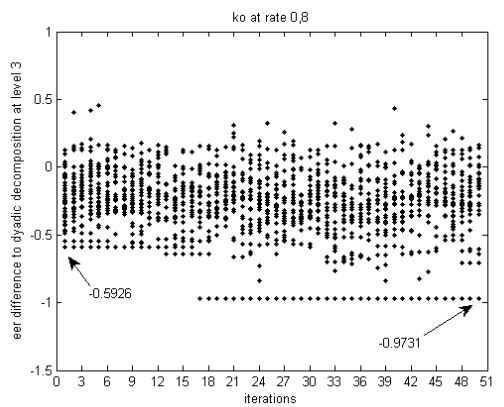

tournament selection

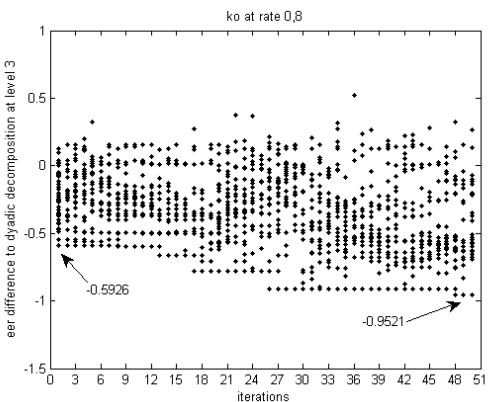

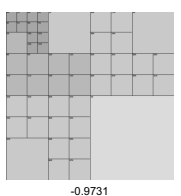
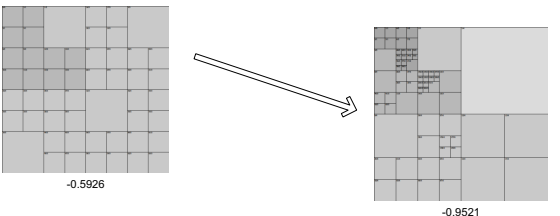

Fig. 1. Evolvement of $w p b$ for Ko et al. recognition @ 0.8 bpp 
9.28, 9.51, 9.40, 9.18, EER(Monro $)=2.24,1.40,1.35,1.36)$, the improvements of 0.97 (Ko@ 0.8bpp,roulette wheel selection) and 0.77 (Monro @ 0.2bpp, roulette wheel selection) can be rated clearly significant.

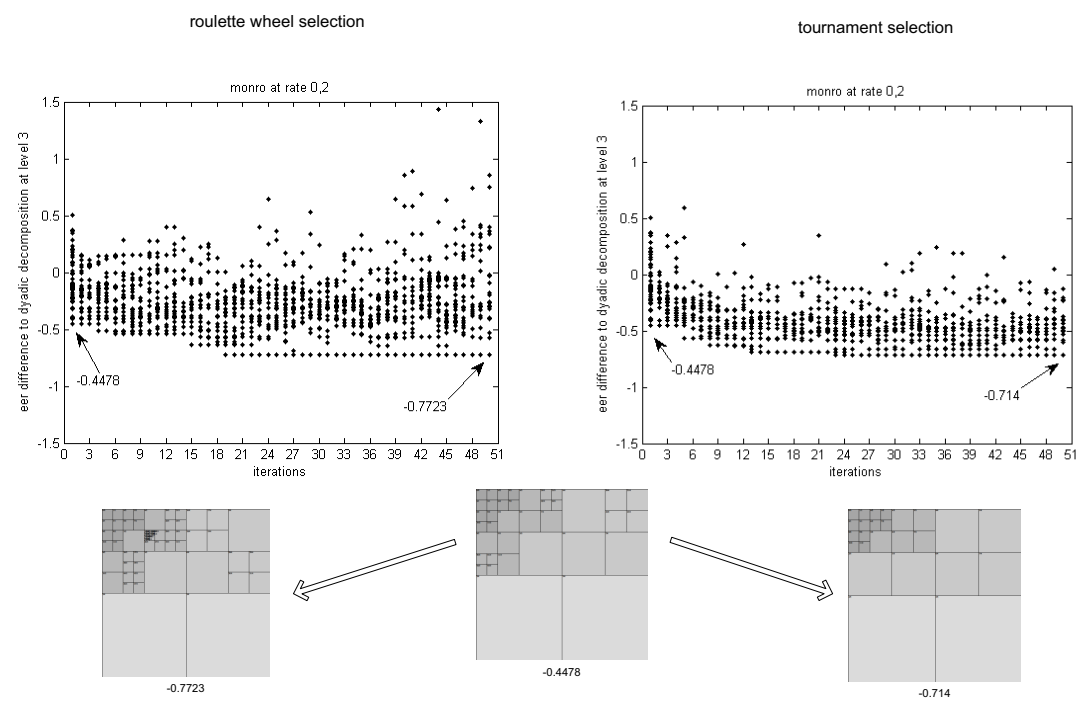

Fig. 2. Evolvement of $w p b$ for Monro et al. @ 0.2 bpp

Table 1 provides the overall view. For each bitrate considered, JPEG2000 Part 2 compression is able to significantly outperform the dyadic JPEG2000 Part 1 scheme. While for the Ko et al. recognition algorithm improvements are distributed rather uniformly over different bitrates, for Monro et al. the case $0.2 \mathrm{bpp}$ shows the highest optimisation potential (EER is reduced from 2.24 to 1.47 !). In all but a single case, roulette wheele selection exhibits superior results to tournament selection.

Table 1. Results of the individual bitrate optimizations (improvements in terms of EER)

\begin{tabular}{l|l|l|l|l} 
Ko et al. & 0.2 bpp & 0.4 bpp & 0.8 bpp & 1.5 bpp \\
\hline Roulette wheel selection & $\mathbf{- 0 . 5 4 0 1}$ & $\mathbf{- 0 . 8 1 5 4}$ & $\mathbf{- 0 . 9 7 3 1}$ & $\mathbf{- 0 . 6 2 3 3}$ \\
Tournament selection & $\mathbf{- 0 . 4 8 1 7}$ & $\mathbf{- 0 . 7 9 4 0}$ & $\mathbf{- 0 . 9 5 2 1}$ & $\mathbf{- 0 . 5 4 4}$ \\
& & & & \\
& 0.2 bpp & 0.4 bpp & 0.8 bpp & 1.5 bpp \\
\hline Monro et al. & $\mathbf{- 0 . 7 7 2 3}$ & $\mathbf{- 0 . 2 5 3 1}$ & $\mathbf{- 0 . 2 6 2 1}$ & $\mathbf{- 0 . 1 0 0 0}$ \\
\hline Roulette wheel selection & $\mathbf{- 0 . 7 1 4 0}$ & $\mathbf{- 0 . 1 5 6 4}$ & $\mathbf{- 0 . 2 3 6 8}$ & $\mathbf{- 0 . 1 4 3 7}$
\end{tabular}

Having seen the potential of optimisation to a single target bitrate, the question arises natually if there exist $w p b$ which outperform the dyadic decomposition for several bitrates (we stick to the four bitrates defined before) - "cross-bitrate optimisation". The answer is "yes", as illustrated in Fig. 3. Figs. 3, a - 3, b show the gain in EER (y-axis) of the top-six $w p b$ individuals for the four target bitrates (x-axis). We clearly see that we succeed with our optimisation, but the improvements are less distinct as compared 
to the optimisation for a single bitrate. For Ko et al. recognition, optimisation with respect to the mean EER gain leads to several $w p b$ which improve over the dyadic case for all bitrates considered (see Fig. 3,a). For the Monro et al. scheme (Fig. 3b), the fitness function involving the standard deviation leads to some uniformly distributed EER gain, however, the amount of achieved gain is not very high (Fig. 3, b).

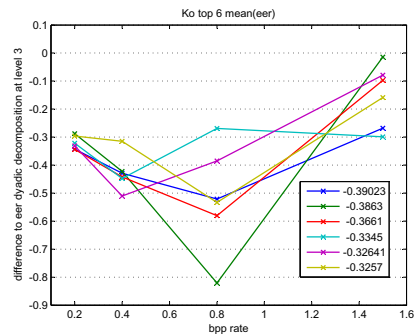

(a) Ko (mean)

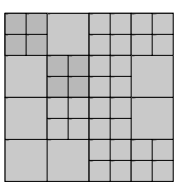

(c) -0.3902

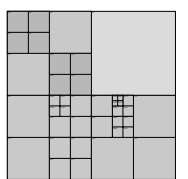

(d) -0.3863

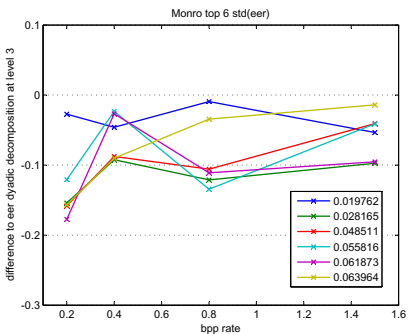

(b) Monro (std)

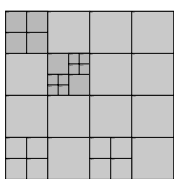

(e) 0.01976

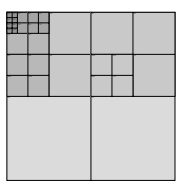

(f) 0.06396

Fig. 3. Cross-bitrate optimisation results

Fig. 3 c - Fig. 3f illustrate some of the evolved $w p b$, the value in the corresponding caption is the cost function value (mean or standard deviation of EER gain). The first two $w p b$ correspond to Fig. 3 a - while they look fairly different overall, at least the LL-band decompsition (top left quater) is identical (which is the case for all but one of those top-six results). The last two $w p b$ correspond to Fig. 3 , $\mathrm{b}$ - they do not exhibit structural similarity among each other nor to the $w p b$ considered before.

The aim of the following investigations is to verify if the behaviour of the optimised $w p b$ generalise to different scenarios. First we look into intra-database verification, i.e., we apply the $w p b$ optimised on the first part of the CASIA V3 Interval dataset for a specific target bitrate to the remaining part of the database using the same bitrate. Consequently, no evolutionary optimisation is involved in the following experiments, the results of which are shown in Table 2 .

Results clearly indicate that the results do not at all generalise. While for some specific settings we still observe significant EER improvements as compared to the JPEG2000 Part 1 dyadic scheme (e.g. Ko et al. with roulette wheel selection @ 0.4bpp or Monro et al. with tournament selection @ 0.2bpp), for most scenarios the EER gain is not significant, in some cases EER even clearly degenerates (e.g. Monro et al. with roulette wheel selection @ $0.2 \mathrm{bpp}$ ).

Table 3 shows the results of the cross-database verification, where the $w p b$ optimised on a part of the CASIA V3 Interval database are applied to a part of the IITD database. As expected (when considering the previous results), results do not generalise as well. 
Table 2. Results of intra-database verification (individual bitrate optimizations, improvements in terms of EER)

\begin{tabular}{l|c|c|c|c} 
Ko et al. & 0.2 bpp & 0.4 bpp & 0.8 bpp & 1.5 bpp \\
\hline Roulette wheel selection & $\mathbf{- 0 . 0 8 7 4}$ & $\mathbf{- 0 . 2 5 2 9}$ & $\mathbf{- 0 . 0 1 4 3}$ & $\mathbf{- 0 . 1 5 4 1}$ \\
Tournament selection & $\mathbf{- 0 . 2 2 8 2}$ & $\mathbf{- 0 . 1 0 1 2}$ & 0.0264 & $\mathbf{- 0 . 0 0 7 5}$ \\
& & & & \\
& 0.2 bpp & 0.4 bpp & 0.8 bpp & 1.5 bpp \\
\hline Monro et al. & 0.3974 & 0.0158 & $\mathbf{- 0 . 0 7 1 1}$ & $\mathbf{- 0 . 0 1 0 5}$ \\
\hline Roulette wheel selection & $\mathbf{- 0 . 1 5 1 4}$ & $\mathbf{- 0 . 0 8 6 7}$ & $\mathbf{- 0 . 1 0 7 4}$ & $\mathbf{- 0 . 0 0 4 1}$
\end{tabular}

Interestingly, at least for roulette wheel selection a sight gain is observed for all bitrates and both recognition algorithms but given the amount of improvement (especiall for the Monro et al. scheme) this seems to be a random phenonemon only.

Table 3. Results of cross-database verification (individual bitrate optimizations, improvements in terms of EER)

\begin{tabular}{l|c|c|c|c} 
Ko et al. , IITD & 0.2 bpp & 0.4 bpp & 0.8 bpp & 1.5 bpp \\
\hline Roulette wheel selection & $\mathbf{- 0 . 2 0 6 3}$ & $\mathbf{- 0 . 1 1 0 3}$ & $\mathbf{- 0 . 3 9 5 1}$ & $\mathbf{- 0 . 1 5 4 1}$ \\
Tournament selection & $\mathbf{- 0 . 3 4 7 1}$ & 0.0414 & $\mathbf{- 0 . 3 5 4 3}$ & $\mathbf{- 0 . 0 0 7 5}$ \\
& & & & \\
& & & \\
Monro et al. , IITD & 0.2 bpp & 0.4 bpp & 0.8 bpp & 1.5 bpp \\
\hline Roulette wheel selection & $\mathbf{- 0 . 3 4 9 4}$ & $\mathbf{- 0 . 0 8 8 8}$ & $\mathbf{- 0 . 0 4 5 1}$ & $\mathbf{- 0 . 1 4 6 1}$ \\
Tournament selection & $\mathbf{- 0 . 1 8 0 6}$ & 0.1846 & 0.0145 & 0.0697
\end{tabular}

Finally, we apply the $w p b$ optimised for one iris recognition scheme using the other scheme. The dataset of the intra-database verification is used, while optimisation has been done on the first part of the CASIA V3 Interval dataset as before. Results are displayed in Table 4 which show that in about half the cases, EER is degenerated.

Table 4. Results of cross-algorithm verification (individual bitrate optimizations, improvements in terms of EER)

\begin{tabular}{l|c|c|c|c} 
Ko verifies Monro & 0.2 bpp & 0.4 bpp & 0.8 bpp & 1.5 bpp \\
\hline Roulette wheel selection & 0.1539 & $\mathbf{- 0 . 1 3 6 0}$ & 0.3710 & 0.0123 \\
Tournament selection & 0.1807 & $\mathbf{- 0 . 7 0 2 9}$ & $\mathbf{- 0 . 1 8 3 3}$ & 0.0416 \\
& & & & \\
Monro verifies Ko & 0.2 bpp & 0.4 bpp & 0.8 bpp & $1.5 b p p$ \\
\hline Roulette wheel selection & 0.2761 & 0.1444 & $\mathbf{- 0 . 0 4 0 6}$ & 0.0623 \\
Tournament selection & $\mathbf{- 0 . 0 1 6 9}$ & 0.1501 & $\mathbf{- 0 . 0 7 1}$ & $\mathbf{- 0 . 0 0 5 0}$
\end{tabular}

\section{Conclusion}

The obtained results show that evolutionary optimisation of wavelet packet subband structures in JPEG2000 with respect to a fixed target bitrate is possible and leads to a 
significant gain in terms of EER as compared to the dyadic JPEG2000 Part 1 decomposition. Also optimisation for a set of bitrates is possible, however, the gain in EER is clearly lower as compared to the individual bitrate optimisation strategy. Our results also show that the computed wavelet packet subband stuctures are highly tuned to the dataset used in the optimisation - results do neither generalise to a different sample database (different capturing conditions) nor to a different subset of the database used for optimisation (different individuals). Furthermore, results are highly specific for the recognition algorithm they have been optimized for and do not at all carry over to a different feature extraction and matching scheme. Thus, the proposed approach can be used for a closed application with a fixed user set - a verification scenario as it is expected at border control with multi-national passports obviously cannot be supported. For such a scenario with dynamically varying user group it is best to stick to the fixed pyramidal decomposition of JPEG2000.

\section{References}

[1] Daugman, J., Downing, C.: Effect of severe image compression on iris recognition performance. IEEE Transactions on Information Forensics and Security 3(1), 52-61 (2008)

[2] Rathgeb, C., Uhl, A., Wild, P.: Iris Recognition: From Segmentation to Template Security. Advances in Information Security, vol. 59. Springer (2013)

[3] Ives, R.W., Broussard, R.P., Kennell, L.R., Soldan, D.L.: Effects of image compression on iris recognition system performance. Journal of Electronic Imaging 17, 011015 (2008), doi:10.1117/1.2891313

[4] Rakshit, S., Monro, D.: An evaluation of image sampling and compression for human iris recognition. IEEE Transactions on Information Forensics and Security 2(3), 605-612 (2007)

[5] Kasaei, S., Deriche, M., Boashash, B.: A novel fingerprint image compression technique using wavelet packets and pyramid lattice vector quantization. IEEE Transactions on Image Processing 12(11), 1365-1378 (2002)

[6] Bradley, J.N., Brislawn, C.M., Hopper, T.: The FBI wavelet/scalar quantization standard for gray-scale fingerprint image compression. In: SPIE Proceedings, Visual Information Processing II, Orlando, FL, USA, vol. 1961, pp. 293-304 (April 1993)

[7] Hämmerle-Uhl, J., Karnutsch, M., Uhl, A.: Recognition impact of JPEG2000 part 2 wavelet packet subband structures in polar iris image compression. In: Zovko-Cihlar, B., Rupp, M., Mecklenbräuker, C. (eds.) Proceedings of the 19th International Conference on Systems, Signals and Image Processing (IWSSIP 2012), pp. 13-16 (2012)

[8] Rajpoot, N.M., Wilson, R.G., Meyer, F.G., Coifman, R.R.: Adaptive wavelet packet basis selection for zerotree image coding. IEEE Transactions on Image Processing 12(12), 1460$1472(2003)$

[9] Stütz, T., Uhl, A.: Efficient and rate-distortion optimal wavelet packet basis selection in JPEG2000. IEEE Transactions on Multimedia 14(2), 264-277 (2012)

[10] Taubman, D., Marcellin, M.: JPEG2000 — Image Compression Fundamentals, Standards and Practice. Kluwer Academic Publishers (2002)

[11] Schell, T., Uhl, A.: Optimization and assessment of wavelet packet decompositions with evolutionary computation. EURASIP Journal on Applied Signal Processing 2003(8), 806813 (2003)

[12] Ko, J.G., Gil, Y.H., Yoo, J.H., Chung, K.I.: A novel and efficient feature extraction method for iris recognition. ETRI Journal 29(3), 399-401 (2007)

[13] Monro, D., Rakshit, S., Zhang, D.: DCT-based iris recognition. IEEE Transactions on Pattern Analysis and Machine Intelligence 29(4), 586-595 (2007) 